\title{
Dapsone Induced Agranulocytosis and Hemolytic Anemia in Immune Thrombocytopenic Purpura
}

\author{
Sandip P. Bartakke ${ }^{1} \cdot$ Abhilasha A. Sampagar ${ }^{2} \cdot$ Nalla Anuraag Reddy ${ }^{2} \cdot$ Ranjit Kangle $^{3}$
}

Received: 29 June 2019 / Accepted: 29 January 2020 / Published online: 5 March 2020

(C) Dr. K C Chaudhuri Foundation 2020

To the Editor: Dapsone is a commonly used second-line drug for immune thrombocytopenia (ITP) in developing countries as it is economical and efficacious [1]. Rarely, dapsone can cause idiosyncratic reaction leading to agranulocytosis [2]. We recently encountered a case who suffered from dapsone induced agranulocytosis (DIA) and severe hemolysis.

A 13-y-old girl was diagnosed as a case of ITP at other center 18 mo ago. She was steroid refractory and put on dapsone $(100 \mathrm{mg} / \mathrm{d})$ for symptomatic bleeding. After $6 \mathrm{wk}$, her platelet counts increased from $12,000 / \mathrm{mm}^{3}$ to $95,000 / \mathrm{mm}^{3}$. However, the other parameters of complete blood counts (CBC) were not monitored. After $1 \mathrm{mo}$, she presented with high grade fever, cough, dyspnea and pallor to our hospital. She was hemodynamically unstable and was admitted in the PICU.

Her CBC showed $\mathrm{Hb}-6.8 \mathrm{~g} / \mathrm{dl}$, WBC $-1000 / \mathrm{mm}^{3}$ with absolute neutrophil count of $40 / \mathrm{mm}^{3}$, platelets $-360,000 / \mathrm{mm}^{3}$, corrected reticulocyte count $-2.6 \%$. Peripheral smear showed polychromasia. Liver function test showed unconjugated hyperbilirubinemia. Chest radiograph revealed left lobe consolidation. Blood culture grew Klebsiella pneumoniae.

Bone marrow examination showed marked suppression of myeloid series and maturation arrest consistent with agranulocytosis. She was treated with granulocyte colony stimulating factor for $6 \mathrm{~d}$ and appropriate antibiotics. She also required mechanical ventilation and inotropic support. After $10 \mathrm{~d}$, her clinical recovery coincided with neutrophil count recovery.

Abhilasha A. Sampagar

docabhilasha@gmail.com; abhilasha.pedia@gmail.com

1 Department of Pediatrics, Bharati Vidyapeeth Medical College and Hospital, Pune, India

2 Department of Pediatrics, JN Medical College, KAHER's University, Belagavi, India

3 Department of Pathology, JN Medical College, KAHER's University, Belagavi, India
Agranulocytosis and hemolytic anemia were attributed to dapsone which was withheld.

Various hematological adverse effects of dapsone have been reported, like hemolysis, methemoglobenemia and pulmonary eosinophilia. Although dapsone has been reported to cause agranulocytosis during the treatment of dermatitis herpetiformis and leprosy, there are no case reports of DIA during the management of ITP [3].

DIA is a rare but catastrophic complication, observed 4-12 wk after the initiation of dapsone [2]. There are no guidelines for the maximum dose of dapsone in pediatric age group, however, a dose of $>100 \mathrm{mg} / \mathrm{d}$ is associated with high risk of toxicity in adults [4]. Hydroxylamine, a metabolite of dapsone is implicated in maturation arrest of neutrophils which leads to agranulocytosis [3]. Although dapsone is known to induce mild hemolysis, in our case, patient developed significant hemolytic anemia.

This case emphasizes that patients receiving dapsone should be regularly monitored for neutropenia and hemolytic anemia.

\section{Compliance with Ethical Standards}

Conflict of Interest None.

\section{References}

1. Patel AP, Patil AS. Dapsone for immune thrombocytopenic purpura in children and adults. Platelets. 2015;26:164-7.

2. Coleman MD. Dapsone-mediated agranulocytosis: risks, possible mechanisms and prevention. Toxicology. 2001;162:53-60.

3. Bhat RM, Radhakrishnan K. A case report of fatal dapsone-induced agranulocytosis in a Indian mid-borderline leprosy patient. Lepr Rev. 2003;74:167-70.

4. Paniker U, Levine N. Dapsone and sulfapyridine. Dermatol Clin. 2001;19:79-86.

Publisher's Note Springer Nature remains neutral with regard to jurisdictional claims in published maps and institutional affiliations. 